\title{
Interactive comment on "Bipolar long-term high temporal resolution broadband measurement system for incoming and outgoing solar UV radiation, and snow UV albedo, at Sodankylä $\left(67^{\circ} \mathrm{N}\right)$ and Marambio (64 S)" by $\mathrm{O}$. Meinander et al.
}

Anonymous Referee \#2

Received and published: 19 February 2016

\section{General comments}

The manuscript of Meinander and others has two main aims: (1) to provide a technical description of UV radiation and albedo measurement stations in the Finnish Arctic (Sodankylä) and maritime Antarctic (Marambio), including an identification of potential errors; and (2) provide a literature review of existing publications (mainly scientific reports, which are presumably not widely available) at one site (Sodankylä). The first part provides a useful scientific reference for potential users of the data and for establishment of similar high quality UV measurement systems elsewhere (although the section

Printer-friendly version

Discussion paper 
on measurement 'challenges' really needs to quantify error sources and provide methods to rectify them). The second part is rather ineffective consisting of a disparate collection of observations and somewhat speculative interpretation.

The paper could be shortened considerably by using more concise language and better structure to avoid the frequent repetition. There are also a couple of places were the language is convoluted and unclear, together with other basic problems of expression such as undefined acronyms.

\section{Specific comments}

1. The Marambio measurements appear to be made in a large container, although this isn't entirely clear. This setup needs a fuller explanation and an assessment of the effect of the container on the measurements through shadowing and impact on wind drift needs to be evaluated.

2. Section 2.5 identifies a number of potential error sources (mistermed as 'challenges'), but doesn't go any further than this. Without quantifying these errors and explaining how to remedy them this section isn't much use to potential users of the data.

3. Section 3 (the second part of the paper) is a rather disparate collection of observations, simplistic analysis and somewhat speculate interpretation which doesn't seem to have clear guiding objectives. In order to understand this section the underpinning data need to be displayed in graphs and tables and the measurement methods, for example how sampling for impurities was conducted, need to be explained. Some similar observations, e.g. daytime hysteresis, have also been made in broadband albedo measurements of snow, but there is no cross referencing to this literature to identify common explanations. Section 3 might be better presented as either a short section ( 1 or 2 paragraphs) just reporting the main findings, or else removed entirely and written up as a full review paper which could provide the required critical evaluation and cross-referencing.

Printer-friendly version

Discussion paper 


\section{Technical corrections}

L18 'large amount of' is vague and can be removed

L24, and L57 and elsewhere in the paper 'for the first time' doesn't need to be stated as it should be obvious that a scientific paper is presenting something new

L24-L28 A very convoluted sentence which doesn't make grammatical sense. Rewrite as two or more sentences, and explain what the 'comparison' is between

L35 and L68-L70 'literature review' implies a critical evaluation of a broad sweep of published material which is not what you do. 'Summary' would be a better term

L36 explain these publications are mainly reports which are not widely available (if that is the case)

L53 RT is not defined

L57-60 Same comment as for L24-L28

L80 don't abbreviate 'figure' and be consistent in this throughout the paper

L93 insert 'a' before 'snow surface'

L103 'September or October' is vague, give the exact date

L173 This is not a good title for the section. 'Error sources and their treatment' would be a more informative title Section 2.5 This section could be written much more concisely

L181 '. . . regions, characterized by. .. '

L182-L183 'low solar elevation' vague, give the exact elevation

L185 'Due to frequent calm or low wind speeds. .' would be a better way to start this line

L187 'really low' vague, give the exact elevations 
L191 when does the sun appear exactly?

L192 again, give the solar elevation

\section{GID}

L198 '. . .maintain clean and free. ..'; '. . . visited only once. ..'

L199-L202; wind appears 3 times as points ii), iv) and v). Isn't wind just a single hazard?

Interactive

comment

L200 what exactly is meant by 'uncomfortable' and is this really relevant to the scientific integrity of the data?

L205 Should this section be called 'Summary of results from previous studies using Sodankylä and Marambio data'

L206-L211 This overview of the paper is repetition and misplaced here. Integrate with section 1

L212-L215 repetition of section 2.3

\section{L217 define 'SZA'}

\section{L234 define ' $A$ '}

Figure 4 only seems to show the UVB sensors and not the UVA sensor. Is there a reason for this?

Figure 5 please provide a legend. The caption should just explain what is shown in the figure, the description of methods used should be moved to the text of the paper.

Interactive comment on Geosci. Instrum. Method. Data Syst. Discuss., doi:10.5194/gi-201531, 2016. 